\title{
Electronic-Positron (Pairino's) Structure of Nuclear Substance (Matter)
}

\author{
Chuvilskaya Tatjana Victorovna \\ Skobeltsyn Institute of Nuclear Physics, Lomonosov Moscow State University, Moscow 119991, Russia
}

\begin{abstract}
Decrease or enlargement of the number of pairinos in nuclei and as free nucleons, pairinos, electrinos, positpinos is a factor of destabilization in the matter and or environment. For the benefit of a electronic-positron structure the representations about an annihilation of the electronic-positron pairs, formation in substance electronic-positron of pairs under activity action of a gamma radiation, electronic and positron character of a beta radiation in nuclei are worked. Terminology: positrino, electrino, pairino. The new mechanism of a physical phenomenon, well explored to the present moment, with the conventional interpretation - mechanism of an annihilation of a free positron on electrons of substance is offered. That is the conventional point of view is those, that during annihilation the electron burns down, disappears or is transmuted into a gamma-quantum. In opinion of the author any facts or the proofs of such interpretation do not exist. The following mechanism therefore is offered: an electron and positron at coming together are discharged by two gamma-quantum and form pairino (unloaded electron - positron state with gravitational mass " $\mathrm{m}$ " about $2 \mathrm{eV}$, with compensation by electric charge, about an electrino by positrino interaction); that is the mechanism "cold" (low, is less than $10 \mathrm{KeV}$, energies) synthesis of pairino. The given mechanism of phenomenon of annihilation is more realistic.
\end{abstract}

Key words: Positrino, electrino, pairino.

\section{Introduction}

The setting of the task consists in revising available physical experience and concepts on a nuclear physics produced approximately within 20 centuries from the moment of discovery of a phenomenon of a radioactivity. As the physical examinations were carried out during a long-lived time slice and on different directions, to the present time the major information material is accumulated which, is represented, requires ordering (or as is spoken, decomposition "on half-glasses").

On the other hand the task on ordering even of all material on a nuclear physics is represented enough cumbersome. But the revising any of the block information, apparently, is already useful in the given direction of common tasks. The engaging of modern personal computers for analysis is necessary.

Corresponding author: Chuvilskaya Tatjana Victorovna, senior scientist, Ph.D., research fields: nuclear physics, nuclear reactions and nuclear isomers. E-mail: tatchuv@anna19.sinp.msu.ru; tatchuv@rambler.ru.
From the logical point of view by a substantiation of falsehood, any of the statement (assertion, judgment) or plurality (collection) of the statements (theory) is the proof of a truth of its negation. For example, the refutation of the theory can be the "deciding" experiment. A design of experiments in such direction is expedient for a fundamental science.

\section{Pairino's Structure of Nuclear Substance}

For the benefit of a electronic-positron structure the representations about an annihilation of the electronic-positron pairs, formation in substance electronic-positron of pairs under activity action of a gamma radiation, electronic and positron character of a beta radiation in nuclei are worked.

\section{New Mechanism of Phenomenon of an Annihilation}

The new mechanism of a physical phenomenon, well explored to the present moment, with the conventional interpretation - mechanism of an 
annihilation of a free positron on electrons of substance is presented [1]. That is the conventional point of view [2-4] is those that during an annihilation the electron burns down, disappears or is transmuted into a gamma-quantum. In opinion of the author, any facts or the proofs of such interpretation do not exist.

The following mechanism therefore is offered: an electron and positron at coming together are discharged by two gamma-quantum and form a pairino $[\mathrm{e}-\mathrm{e}+]$ (unloaded electron - positron state with gravitational mass " $\mathrm{m}$ " $2 \mathrm{eV}$, with compensation by electric charge, about an electrino [e-] by positrino $\left[\mathrm{e}^{+}\right]$ interaction); that is the mechanism cold (low, is less $10 \mathrm{keV}$, energy) synthesis of pairino. The given mechanism of phenomenon of an annihilation is more realistic.

\section{Experience on Existence of a Neutrino}

Two types of experience on detection a neutrino [5] now are known [6,7]:

1-st type - with an antineutrino from reactor,

2 type - with a solar neutrino.

The reaction is in first case explored:

$$
\mathrm{p}+\mathrm{x} \rightarrow \mathrm{n}+\mathrm{e}^{+}
$$

where, $x$-antineutrino. In the second case of a nucleus $37 \mathrm{Cl}$ or $71 \mathrm{Ga}$ capture a solar neutrino and form accordingly $37 \mathrm{Ar}(\mathrm{T} 1 / 2=35$ of days $)$ or $71 \mathrm{Ge}(\mathrm{T} 1 / 2=$ 11 of days), i.e. the reaction is explored:

$$
\mathrm{n}+\mathrm{z} \rightarrow \mathrm{p}+\mathrm{e}^{-}
$$

where, z-neutrino.

In both cases there is an infringement of conservation laws of mass and charge, as not the material particle cancels or forms a charge and plus still forms a charged particle. Besides there is an ambiguity of such transmutations, they can be exchanged with reactions of absorption of pairino [e-e+]:

$$
\begin{aligned}
& \mathrm{p}+\left[\mathrm{e}^{-}\right] \rightarrow \mathrm{n} \\
& \mathrm{n}+\left[\mathrm{e}^{+}\right] \rightarrow \mathrm{p}
\end{aligned}
$$

In both cases the background measurements by detectors of large volume will be carried out.

\section{Beta Decay}

The most spread expressions of record of conservation laws of mass and charge in three views of a beta decay are:

$$
\begin{gathered}
M(A, Z)>M(A, Z+1)+m \quad\left(\beta^{-}\right) \\
M(A, Z)>M(A, Z-1)+m^{+} \quad\left(\beta^{+}\right) \\
M(A, Z)+m>M(A, Z-1) \quad(K-\text { capture })
\end{gathered}
$$

where, $M$ and $m$ are the mass accordingly of nuclei and $\mathrm{e}^{+}$. Or through masses of neutral atoms the energy of $\beta^{-}$decay $E_{\beta}$ expresses:

$$
\begin{gathered}
\mathrm{E}_{\beta}=[\mathrm{M}(\mathrm{A}, \mathrm{Z})-\mathrm{M}(\mathrm{A}, \mathrm{Z}+1)] \mathrm{c}^{2} \\
\left(\beta^{+}\right) \mathrm{E}=[\mathrm{M}(\mathrm{A}, \mathrm{Z})-\mathrm{M}(\mathrm{A}, \mathrm{Z}-1)] \mathrm{c}^{2}-2 \mathrm{~m}_{0} \mathrm{c}^{2} \\
(\mathrm{e}) \mathrm{E}=[\mathrm{M}(\mathrm{A}, \mathrm{Z})-\mathrm{M}(\mathrm{A}, \mathrm{Z}-1)] \mathrm{c}^{2}
\end{gathered}
$$

It is represented expedient to note requirements of a $\beta$ decay through binding energies of an initial and terminating nuclei:

$$
\begin{aligned}
& E_{b}(A, Z+1)-E_{b}(A, Z)>\left(M_{p}-M_{n}\right) c^{2}+m_{0} c^{2} 1(11) \\
& E_{b}(A, Z-1)-E_{b}(A, Z)>\left(M_{n}-M_{p}\right) c^{2}+m_{0}{ }^{+} c^{2} 2(12)
\end{aligned}
$$

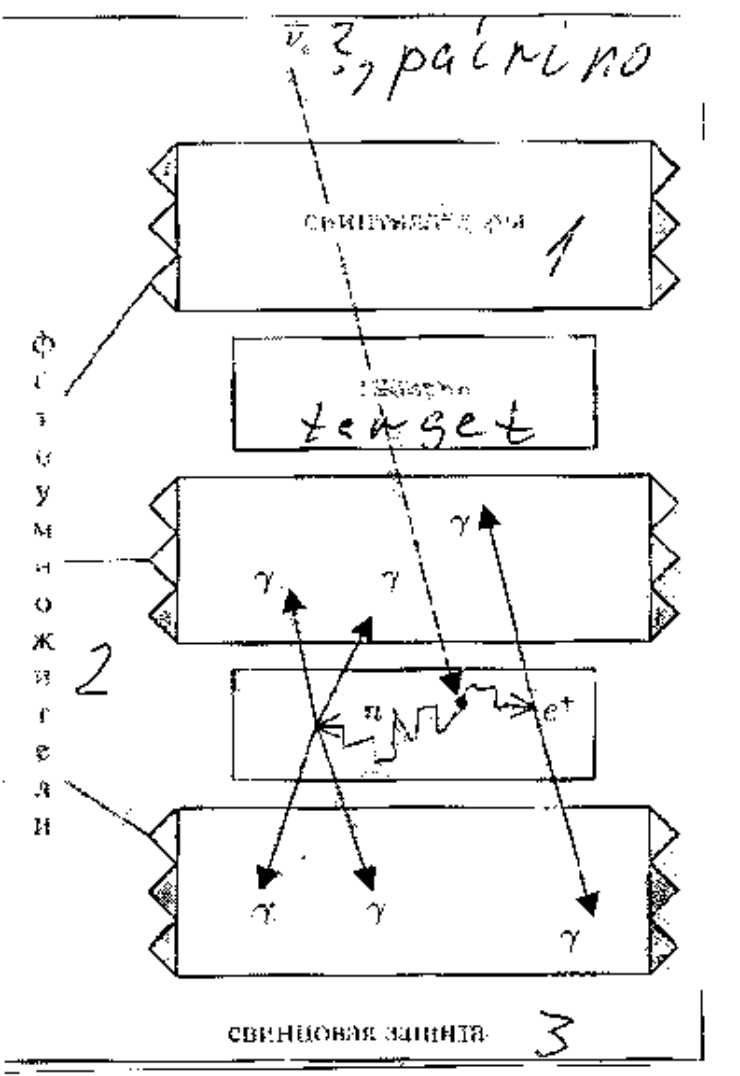

Fig. 1 Raens and Coen experiments 1-scintillators, 2photomultipliers and 3 -lead radiation protection. 
$E_{b}(A, Z-1)-E_{b}(A, Z)>\left(M_{n}-M_{p}\right) c^{2}-m_{0} c^{2} 3(13)$ Requirements whence follow:

$$
\begin{aligned}
& \text { 1. }\left(M_{n}-M_{p}\right) c^{2}>m_{0} c^{2} h n \rightarrow h p \\
& \quad \text { So-called } \rightarrow \text { etc. } \\
& \text { 2. }\left(M_{p}-M_{n}\right) c^{2}>m_{0}{ }^{+} c^{2} \text { etc. } \rightarrow \ln \\
& \text { 3. }\left(M_{n}-M_{p}\right) c^{2}<m_{0} c^{2} l p \rightarrow 1
\end{aligned}
$$

Etc. $\rightarrow$ so-called, where, ln-light neutron, lp-light proton, so-called - heavy neutron, etc. - heavy proton.

As to a law of conservation of momentum in a neutrino-less $\beta$ decay the mechanism of decay is other, at first, are possible (probable) (for example statistical mechanisms in nuclear reactions or Mossbauer effect), and secondly, it is impossible to compare a start of an $\alpha$-particle or $\gamma$-quantum from a nucleus to a start of an electron or positron by virtue of two-gradual of formation of an electron or positron as against one-step character of a start of an $\alpha$-particle or $\gamma$-quantum.

\section{Binding Energy}

The binding energy of a nucleus is a concept, defining nucleus as a system of nucleons as against the simple total of masses of nucleons (free). The alternate approach consists in understanding of a binding energy in considering (counting) nuclear mass not smaller on quantity of a binding energy of the total of masses of free nucleons, and stationary value for a given nucleus. So it is needed to consider mass of a nucleon as the total of mass " $m$ " and energy (kinetic and electromagnetic radiation). Thus aggregate mass of a nucleon is various in a free state and in various nuclei because of an opportunity of accumulation and rebound by a nucleon of the same quantity " $\mathrm{m}$ " of a kinetic energy and energy of an electromagnetic radiation. The energy of an electromagnetic radiation strengthens a mechanical motion, and the mechanical motion strengthens an opportunity of an electromagnetic radiation at acceleration and inhibiting action.

The value " $m$ " of nucleons is different in various nuclei and in one kernel - from here probability processes in nuclei taking place be and in substance the half-life periods accordingly are various, i.e. there are average parameters.

The basic deduction from concept of a binding energy - " $m$ " of micro-particles does not vary (without special conditions), and the total " $\mathrm{m}$ " + energy (kinetic and electromagnetic radiation) can change in various systems with a nucleus.

\section{About Higgs, W, Z-Bosons and $u, d$, Top-Quarks}

Agrees [8, 9] a problem number 1 of the high-energy physics is searches of Higgs particles.. The heavy quarks - c, b, t, $\tau$-leptons, gluons, W, Z-bosons in basic are unclosed and are investigated in experiments on colliding beams of particles [9].

The accelerating accumulative installations with colliding beams term as colliders. Agrees [9], without infringement of CP-invariance, the Universe at early stages of the evolution could not create baryon asymmetry: in it (her) there would be an equal number of protons and antiprotons, electrons and positrons; all of them as a result of an annihilation would turn to quantum and neutrino, and we would not be.

From the point of view of an alternate nuclear physics converts on itself attention that fact, that a unique (sole) stable particle (microscopic object) is the electron, which appears at the end of any chain of decay of partial object [10]. The electron is the most realistic microscopic object and is present at all super-modern systematics of fundamental particles. From the point of view of an alternate nuclear physics of the facts and proofs of any transmutations of an electron, his (its) birth and the erasure is not present even at high energies. The processes of decay of an electron on any component are not known. That is, apparently, is true by partial now it is possible to consider (count) an electron. It (he) has least gravitational mass, up to energies $10^{12} \mathrm{eV}$ are stable. The electron has physical properties of acceleration and inhibiting action, inertial motion. Electron both in 
free state, and into any bound system of a type the atom, nucleus is capable to radiate and to immerse an electromagnetic radiation in a wide range of energies of a quantum - from thermal, wireless, light up to a strong $\gamma$-quantum, is capable to radiate an ionization continuum (synchrotron radiation).

As to a high-energy physics on modern accelerators, any chains of decay [4] of microscopic objects such as:

$$
\begin{aligned}
& \mathrm{ud} \rightarrow \mathrm{W}^{+} \\
& \mathrm{ud} \rightarrow \mathrm{W}^{-}
\end{aligned}
$$

where, W - bosons generated at decay of system a quark an antiquark, which enter a composition of nucleons and are exhibited at collision a proton antiproton on colliders, further break up and the chains of decays of a type are observed: $\mathrm{W}^{-} \rightarrow \mathrm{e}^{-} v_{\mathrm{e}}$, $\mathrm{W}^{+} \rightarrow \mathrm{e}^{+} v_{\mathrm{e}} \cdot \mathrm{Z}_{0}$ the boson breaks up $\mathrm{Z}_{0} \rightarrow \mathrm{e}^{+} \mathrm{e}^{-}$and so on.

That is in any experiments of a similar type the usual partial microscopic objects of a type an electron and positron are filed. The object as neutrino is registered in responses of its interaction with substance and it can be exchanged on pairino, that gives the similar terminating effect (result) on registered yield of the reaction.

But pairino is more realistic object. Apparently, at collisions of high energies $\mathrm{pp}^{-}, \mathrm{pp}, \mathrm{e}^{+} \mathrm{e}-$ strong takes place fragmentation of protons on gravitational mass, number of pairinos, component protons and the recording of various channels, including rare fragmentation is possible(probable). Concerning electronic-positron collisions, if not to expect formation or erasure of gravitational mass at an electron and positron, processes of a type: $\mathrm{e}^{+} \mathrm{e}^{-} \rightarrow Z^{0}$ $\mathrm{H}$, where $\mathrm{H}$ - the Higgs particle with expected energy $200 \mathrm{GeV}$ and not by leptonic character of decay is a clot of an electromagnetic radiation, which can have color and charging asymmetry.

At higher energies of proton colliders it is possible to expect the fragmentation of a combined type on gravitational mass and electromagnetic radiation. These transient states will be discharged probably and to be charged during the evolution.
The confinement of free quarks [11] defines (determines) property of quark stability of a core of a nucleon down to high energies.

From the point of view of an alternate nuclear physics, electrons and positrons as the stable partial objects can differ on gravitational mass and electric charge among themselves, though the quantity $\mathrm{e} / \mathrm{m}$ for a mobile electron in particular sense of precision is a constant on definition of the concept of an electron. Any plurality of electrons and positrons with a charge which is distinct from average value $\mathrm{e}=1.60217733$ (49) $\times 10^{-19} \mathrm{C}$ thus is supposed.

It is a problem of the future - about compensation a charge of pairino, generated during an annihilation. To that the charge completely or partially unloaded, some loaded of pairino is equal? What such is compensation electric charge at a partial level? There is unclosed a problem on recording of pairino and definition of its form-factor.

Agrees [11] all stable substance consists of particles of a first-generation: $\mathrm{e}^{-}, v_{\mathrm{e}}, \mathrm{u}, \mathrm{d}$ - accordingly an electron, neutrino electronic, two quarks - theory Gell-Mann - Nishigina. The same is concerned of the standart model [7]. The given systematics of fundamental particles from the point of view of an alternate nuclear physics is enough integrated in comparison with a electron-positron structure of substance.

As the quark is approximately third of a nucleon, and almost completely charged pairino - thousand part, the degree of stability of a nucleon can be defined(determined) by much more number of the fragmentations by number of pairinos. Included in a composition of a nucleon of pairinos have function from completely, is partial by charged up to unloaded, that is interval of aggregate masses of pairinos ("m" + E) - from $1 \mathrm{MeV}$ up to $2 \mathrm{eV}$ and according to their number from $10^{3}$ up to $10^{9}$.

Quark fragmentation on a charge at collision of nucleons of high energies is function of charging of major number of pairinos, which component nucleons 
and revolutionized probably defines (determines) during response with an opportunity of formation of top-quarks.

Thus alternate nuclear physics negates on sectional stage of a high-energy physics opportunity of birth and erasure of an electron during birth of pairs at inhibiting action of a gamma-quantum and, accordingly, annihilation with positron, and also his (its) decay on component. From positions of an alternate nuclear physics electrons differ in mass of gravitation, energy of radiation, kinetic energy and electric charge at a partial level. The build-up of systems from electrons, positrons supposes, taking into account methods of direct observation, various models and theories on interactions of systems and opportunity of their integrating.

Thus all stable substance consists of particles (corpuscles, microscopic objects) and radiation (photon, quantum): an electron, positron, pairino, electromagnetic radiation.

In connection with above-stated the carrying out of experiments on mini-colliders such as e- $\mathrm{p}, \mathrm{e}^{-},{ }^{4,6,8} \mathrm{He}$ is represented interesting, as any sectional on cold of fragmentation, the neutralizations, except for formation of pairinos in an electron - positron annihilation are unknown.

Besides the carrying out of such experiments is useful from the point of view of a lacing of energies, sectional in a wide range of colliding objects, then joint. It is possible to expect observation of new effects on outputs(exits) of microscopic objects. It also will allow to attract major number of nuclear physicist for the solution of problems on development of a procedure of observation of microscopic objects with detailed elaboration of process in space and time, will enable to mark an electron. The key restrictions for these purposes do not exist. In the future the electrons will collapse and to be synthesized.

\section{References}

[1] Chuvilskaya, T. V. 2015. "Electronic-Positron Structure of Nuclear Substance." In Proceedings of 65 international conference Nucleus S. 270.

[2] Groshev, L. V. and Shapiro, I. S. 1952. "Spectroscopy of the Atomic Nuclei ( in Russian)." Moscow. 42-4.

[3] Siegbahn, K. 1969. "Alpha-, Beta- and Gamma-Ray Spectroscopy (in Russian)." "Atomizdat" Moscow. 4: 254-66.

[4] Djelepov, B. S. and Shestopalova, S. A. 1980. "The Nuclear-Spectroscopic Normals (in Russian)" Moscow-atomizdat. 92-107.

[5] Blatt, J. M. and Weisskopf, V. F. 1954. "Theoretical Nuclear Physics (in Russian)." "IL"-Moscow.525.

[6] Vlasov, N. A. 1971. "The Neutrons (in Russian)." $r$ "Nauka"-Moscow. 33-4.

[7] Ishkhanov, B. S., Kapitonov, I. M. and Yudin, N. P. 2007. "The Particles and Atomic Nuclei." Textbook (in Russian) Moscow. 90-2.

[8] Ginzburg, V. L. 1999. "What a Problems of Physics are Important (in Russian)." UFN 169 (4): 419-41.

[9] Okun, L. B. 1998. "Modern Status of Elementary Particles Physics (in Russian)." UFN 168 (6): 625-9.

[10] Rakobolskaya, I. V. 1981. "A Nuclear Physics." Textbook (in Russian) Publisher of Moscow State University. 220-34.

[11] Clapdor-Klaingrothaus, H. V. and Staudt, A. A. 1997. "A not Accelerating Elementary Particle Physics "(in Russian ) Publisher "Nauka", Moscow. 3-528. 